\title{
Adaptive Robust Video Broadcast via Satellite
}

\author{
M. Altaf ${ }^{1}$. F. Ali' ${ }^{2}$ N. Qadri ${ }^{1}$. M. Ghanbari ${ }^{3}$. Sandra E. Dudley ${ }^{4}$ \\ ${ }^{1}$ COMSATS Institute of Information Technology, Wah, Pakistan, mohammadaltaf@gmail.com, nadianqadri@googlemail.com \\ ${ }^{2}$ COMSATS Institute of Information Technology, Attock, Pakistan, farman marwat@ciit-attock.edu.pk \\ ${ }^{3}$ University of Tehran, Tehran, Iran, ghan@essex.ac.uk \\ ${ }^{4}$ London South Bank University, UK, dudleyms@Isbu.ac.uk
}

\begin{abstract}
With increasing demand for multimedia content over channels with limited bandwidth and heavy packet losses, higher coding efficiency and stronger error resiliency is required more than ever before. Both the coding efficiency and error resiliency are two opposing processes that require appropriate balancing. On the source encoding side the video encoder H.264/AVC can provide higher compression with strong error resiliency, while on the channel error correction coding side the raptor code has proven its effectiveness, with only modest overhead required for the recovery of lost data. This paper compares the efficiency and overhead of both the raptor codes and the error resiliency techniques of video standards so that both can be balanced for better compression and quality. The result is also improved by confining the robust stream to the period of poor channel conditions by adaptively switching between the video streams using switching frames introduced in H.264/AVC. In this case the video stream is initially transmitted without error resiliency assuming the channel to be completely error free, and then the robustness is increased based on the channel conditions and/or user demand. The results showed that although switching can increase the peak signal to noise ratio in the presence of losses but at the same time its excessive repetition can be irritating to the viewers. Therefore to evaluate the perceptual quality of the video streams and to find the optimum number of switching during a session, these streams were scored by different viewers for quality of enhancement. The results of the proposed scheme show an increase of 3 to $4 \mathrm{~dB}$ in peak signal to noise ratio with acceptable quality of enhancement.
\end{abstract}

Keywords Digital Video Broadcast · Error Resiliency · H.264/AVC · Raptor Codes

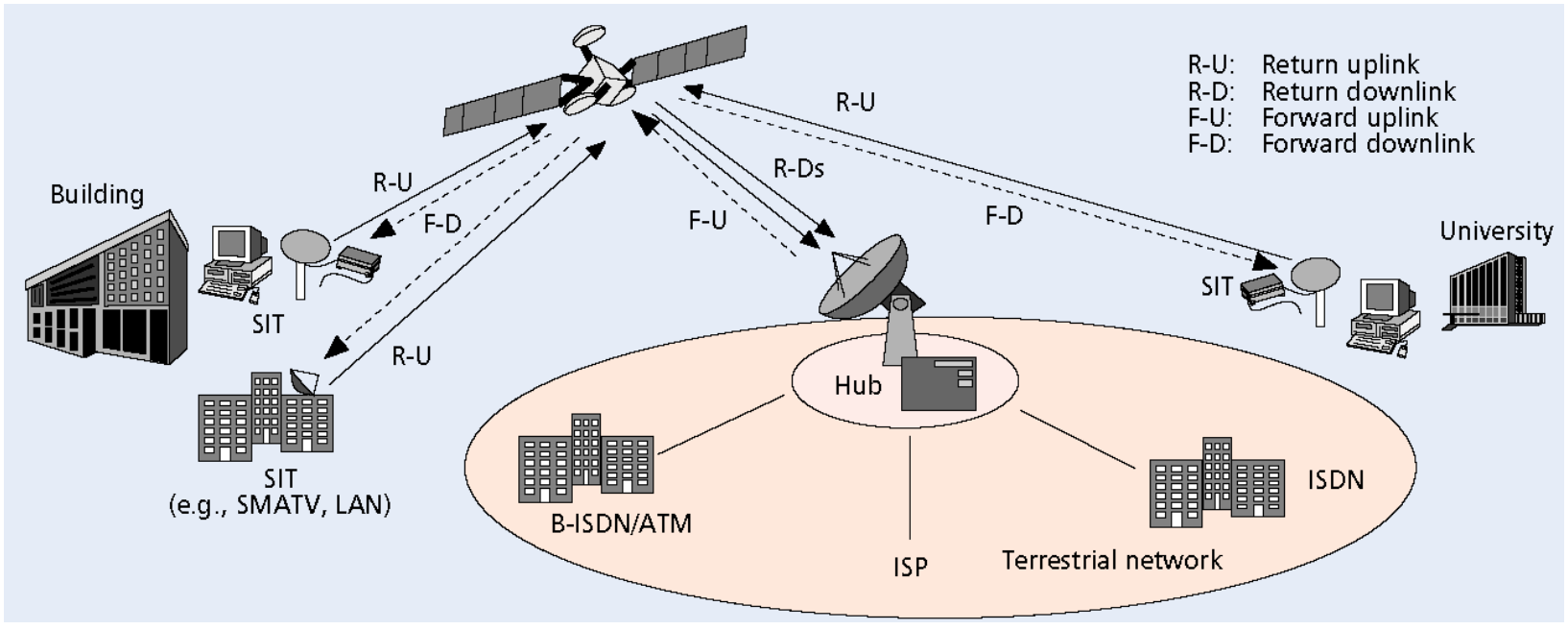

Fig. 1 Interactive channel for multimedia satellite networks [3]

\section{Introduction}

Geostationary (GEO) satellite networks play an important role in the transmission of services such as TV broadcasting, broadband internet, voice and text messaging. Satellite broadcasting has become a preferred medium 
for Digital Video Broadcasting (DVB) across Europe as well as other continents [1]. Based on the DVB-S (-via Satellite) a Uni-Directional Link Routing (UDLR) standard using low bandwidth terrestrial return link and the DVBRCS(-Return Channel Satellite) providing fully bidirectional satellite architecture is specified [2]. The development of DVB-RCS specification began in 1997 and was approved as a European Telecommunications Standards Institute (ETSI) specification in 2000 [1]. The DVB-RCS consists of a forward channel based on DVB/MPEG2 data format and a return link using Multi Frequency Time Division Multiple Access (MF-TDMA) scheme, thus allowing a two way exchange of data [3]. The standard gives complete mechanism for different communication applications ranging from internet to voice over IP and video conferencing via satellite, with end to end quality of service [38]. However the implementation of physical layer interoperability for multi vendor network was hindered due to QoS policies [39]. The QoS was improved in the next generation of the DVB-RCS called DVB-RCS2.

The DVB-RCS concept provided by Jason Neale [3] is shown in Fig. 1 and is described as an Interactive Channel for Multimedia Satellite Networks (ICMSN). The ICMSN return channel provides a direct satellite return channel capacity ranging from $144 \mathrm{Kbps}$ to $2.048 \mathrm{Mbps}$, and a maximum forward data rate up to $45 \mathrm{M} \mathrm{b} / \mathrm{s}$.

Like other wireless channels, satellite links are also error prone and time variant [4]. Robust transmission with adaptive coding modulation (ACM) is introduced in DVB-S2 with an enhancement introduced by Gardikis [5]. These are channel coding techniques used to create streams resilient to channel shadowing and fading [6]. Conversely an approach such as forward error correction (FEC) is used for error correction, but FEC alone is not sufficient to provide full reliability and can be improved by using automatic repeat request [4]. However this may lead to an increased number of feedback information and retransmissions. Moreover, regarding the video transmission that is bounded by real-time constraints, retransmission of lost packets is not a feasible solution. But, as the video coding exploits temporal redundancy through motion estimation between frames [7], the loss of a single packet can cause drift between the encoders and decoders view of the compressed stream. Therefore a suitable choice of source coding robustness technique or FEC technique is required to conceal the effect of lost packets or recover the lost data without the need for retransmissions. Fortunately, in the H.264/Advanced Video Codec (AVC) a range of application-layer error resiliency measures have been pro- vided for wireless communication [8]. The error source coding solutions manage the problem of transmission errors. However, increased error robustness and high compression are two opposing concepts; increasing one may decrease the other [9].

Similar phenomena can also be observed in channel coding for error correction. For example, FEC is an alternative method of detecting and correcting errors but is also based on the redundancy of data. However rateless codes (e.g. Raptor codes) only require slightly more symbols than the number of source symbols for successful recovery [10]. When designed properly, a raptor code can achieve a constant per-symbol encoding and decoding cost with almost zero overhead and a space proportional to the number of packets of the total data [11]. These codes are based on Luby Transform (LT) codes [12] concatenated with a fixed rate channel code and are the most successful rateless codes [11]. For these reasons these codes were adopted by the communication protocols such as 3GPP [13], global IPTV standard DVB Project [14] and Multimedia Broadcast Multicast Services (MBMS) service [13].

To increase the service robustness in mobile networks [15][16][17], raptor codes have been assessed with Scalable extension of H.264. Similarly the performance comparison between Reed-Solomon code as the FEC scheme and a non-scalable video codec is discussed in [18][19]. Comparison of a scalable multiple description coding (MDC) and raptor code for the H.264/SVC (Scalable Video Coding) is presented in [20]. The experimental results show that scalable MDC is generally preferable at the low redundancy rates and long average burst lengths, while FEC using raptor code is favorable in case of high redundancy rates and channels with short average burst lengths for the resilient delivery of scalable video [20]. With the growing interest in Fountain codes, the unequal error protection with these codes has been addressed by several researchers. This usually allows for an improved overall system performance over equal error protection schemes [14].

To the best of our knowledge no previous research has been conducted that compares the robust video source coding with the rateless raptor codes for video streaming in a packet loss scenario as shown in Fig. 1 and switching between them. Although switching between streams with different level of protection for wireless channel is studied in [35]. But this work is limited to switching between streams with sources robustness only without discussing switching between streams protected with source and channel robustness. Another implementation of the stream switching introduced by windows media is the intelligent streaming [36]. In this case the video is encoded at different bitrates 
and hence different qualities without differentiating these streams on the basis of error robustness. The stream is selected as per the available bandwidth of the user. Similar approach is used by RealMedia called SureStream [37], but the switching in this case is also based on the available network resources. Appropriate quality of stream is selected from a pool of multiple bitrate streams based on the available bandwidth. These and other similar applications do not select streams based on packet loss and do not switch to streams protected with different source robustness algorithm or channel error correction codes. This paper discusses in detail the efficiency of raptor encoding on video streaming when the overhead incurred due to redundancy is compensated by an increase in compression of the encoded video stream. Similarly the video is also encoded with different error robustness techniques and the best one in terms of quality versus packet loss rate is compared with that of the raptor encoding. In the second step, the stronger error correction streams are limited to the periods of poor channel condition by switching between the streams and hence improving the average quality of the overall streaming session.

In the proposed method, the video is initially encoded without applying any error resiliency or FEC, assuming the channel to be error-free. In this case the reconstructed video is of better quality than the robust stream versions at the same data rate because there is no coding overhead arising from error resiliency. At times when channel conditions are poor, with a high packet loss rate, the video stream encoded with higher robustness is initiated. Whilst when the channel conditions are favorable, it is possible to switch back to video stream with a reduced level of error resiliency and higher video quality. Switching between streams with different levels of robustness can be initiated by the user or automatically using the return channel in DVB-RCS scenario. The simulation results show an improvement of 3-4 $\mathrm{dB}$ in the quality for the scheme with transition between the streams, for both random and burst packet losses compared to using error resiliency throughout the session.

Although the proposed technique resulted in good peak signal to noise ratio (PSNR) but at the same time increased number of switching and continuous changes in the quality of video may result in decreased quality of enhancement (QoE). QoE is the perceptual quality of the video that may vary from viewer to viewer and scene to scene. In order to decide the optimum number of switching, different video streams with different number of switching were shown to different viewers. The viewers were asked to score the quality of each stream on a scale of 1 to 5 with 5 the highest and 1 the lowest quality. The results showed that an increase in PSNR doesn't mean an increase in the perceptual quality as for as stream switching is concerned. Therefore number of switching between the streams is required to be limited to an optimum value. The results show that an acceptable quality of perception is achieved if next switching is allowed at least 1.5 minutes after the previous switching or in other words in a 60 minutes long video, the switching should not occur more than 40 times.

The rest of the paper is organized as follows; error resiliency techniques of the H.264 along with error concealments techniques and introduction to the raptor codes are given in section 2. Section 3 discusses the assessment of source and raptor coding error resiliency techniques towards isolated and burst errors. The results of adaptive switching between streams with different resilience schemes confining robust video stream to poor channel conditions is given in section 4. This section also discusses the quality of enhancement in detail. Finally section 5 provided the authors concluding remarks, supporting the findings in [35] for mobile wireless channels.

\section{Error Resiliency in Source and Raptor Codes}

H.264 has combined the error resilience schemes of previous coders along with some new techniques [21], included in the video coding layer. The techniques that were also present in previous standards like H.261, H.262, MPEG-1 and MPEG-2 are intra macro block (MB) or intra pictures, picture segmentation (slices or Group of Blocks), data partitioning and reference picture selection. Whilst switching pictures, parameter sets, Flexible Macroblock Ordering (FMO) and Redundant Slices (RS) are newly introduced or differently coded error resiliency tools [21].

Slicing is the division of a picture into portions (slices), whose size can be as small as one macro block and as large as a whole picture arranging macro blocks in raster scan order. Intra-prediction across the slice boundaries is not permitted [22], making a slice a self-contained unit that can be decoded independently without referring to neighboring slices of the frame thus it can prevent error propagation [23].

For FMO, compressed frame data is normally split into a number of slices each consisting of a set of MBs, but are arranged in different order than the raster scan order [21]. Due to the different arrangements of MBs, the possible 
errors are scattered across the whole frame to avoid its accumulation in a smaller area. In this way the distance between the correctly recovered block and the erroneous block is reduced, reducing the distortion in recovered blocks [9].

Data partitioning in H.264/AVC separates the compressed bitstream into: A) configuration data and motion vectors; B) intra-coded transform coefficients; and C) inter-coded coefficients. The data form A, B, and C partitions which are packetized as separate network abstraction layer units with different degrees of importance; partition $\mathrm{A}$ being the most important and partition $\mathrm{C}$ being the least and hence are unequally protected.

The insertion of intra coded MBs into inter-frames allow temporal error propagation to be blocked if matching MBs in a previous frame are lost. Intra refresh through periodic insertion of intra (I-) frames with all MBs encoded through spatial reference is the most common method of limiting error propagation. However, I-frames cause periodic increases in the data rate when encoding at a variable bit rate.

Switching frames are new types of predictive frames and can be reconstructed from different reference frames. Switching frames can be used for stream switching, splicing, random access, fast forward, fast backward, and error resilience/recovery [24].

This brief review by no means exhausts the error-resilience facilities in H.264; for a complete discussion of these techniques and its concealment readers are referred to [9]. In H.264 error concealment is a non-normative feature and is not within the scope of the standard but the standard defines the behavior of the decoder in the presence of errors. Two error concealment algorithms are available in the H.264 test model. The 'Boundary matching' and weighted pixel value averaging for intra pictures. The former uses vectors for inter pictures [8], in which the motion vectors of correctly received slices (or prior concealed slices) are used in boundary matching motion vector recovery. In the latter case, the weighted pixel value averaging for intra pictures, in which it is also possible to select the intra coded frame method of spatial interpolation, provide smooth and consistent edges at an increased computational cost through weighted pixel-value averaging. Interested researchers are referred to [25][26].

In channel error correction coding, raptor (IETF RFC 5053) codes are the most efficient fountain codes [27], with efficient linear time encoding and decoding algorithms. Raptor codes were introduced by Shokrollahi [11] by concatenating a fixed rate channel code with an LT code [12]. Raptor codes have large degrees of freedom in parameter choice [28] having performances comparable to maximum distance separable codes with only slightly more symbols required than the number of source symbols for successful recovery [10]. Raptor codes pre-encode the source symbols using a fixed length block code like Low Density Parity Check Code (LDPC) as a pre-code [29], and then encode these new symbols with an LT code using a suitable degree distribution. The main advantage is that, for correct decoding, it is no longer necessary that the LT decoding succeeds for all the symbols. Thus, it is possible to use a simpler degree distribution that does not recover all the symbols, but increases the speed of the decoding process [30]. A very good comparison of the overhead versus error probability and loss tolerance are shown for the LT codes [31] and raptor codes [32][33][34].

\section{Assessment of Source and Raptor Coding Error Resiliency Techniques}

In this section the relative merits of the error resiliency techniques are assessed, prior to its selection for use with robust video streaming. The HDTV (High Definition TV) sequence Rush hour is used for the simulation purposes. The video sequence was encoded using different error resiliency techniques as in [35] along with different bitrates for transmission with raptor encoding. Transmission of these video streams was simulated with different percentage of random and burst length losses and the results are discussed in the sub sections below. The following source coding robustness techniques are used.

1. Forcing random intra macro blocks in all frames including predictive frames, denoted by int-mb in Fig. 2.

2. Using different number of slices per frame e.g. three slices per frame represented by slice3, eight slices per frame by slice 8 etc as shown in Figs. 2 and 3.

3. Flexible Macro Block Ordering (FMO) for two slice groups, fmo2 and fmo8 etc in Figs. 2 and 3 . In this 
case checker board FMO is used due to the reason that it has better error resiliency compared to other types of FMOs [35].

4. Random Intra MBs were forced along with at least three slices per frame denoted by slice-intmb in Fig. 2.

5. Forcing Intra MB into flexible macro block ordering denoted by fmo-intmb in Fig. 2, checkerboard FMO with two slice groups was used.

3.1 Response of source robustness to random isolated losses

These techniques are compared against a stream without any error resiliency (no- res) for random losses and shown in Fig. 2. It is clear that the quality of the stream without any protection is inferior to other protected streams except when there is no packet loss, since then the lack of coding overhead for an equivalent data rate results in better quality.

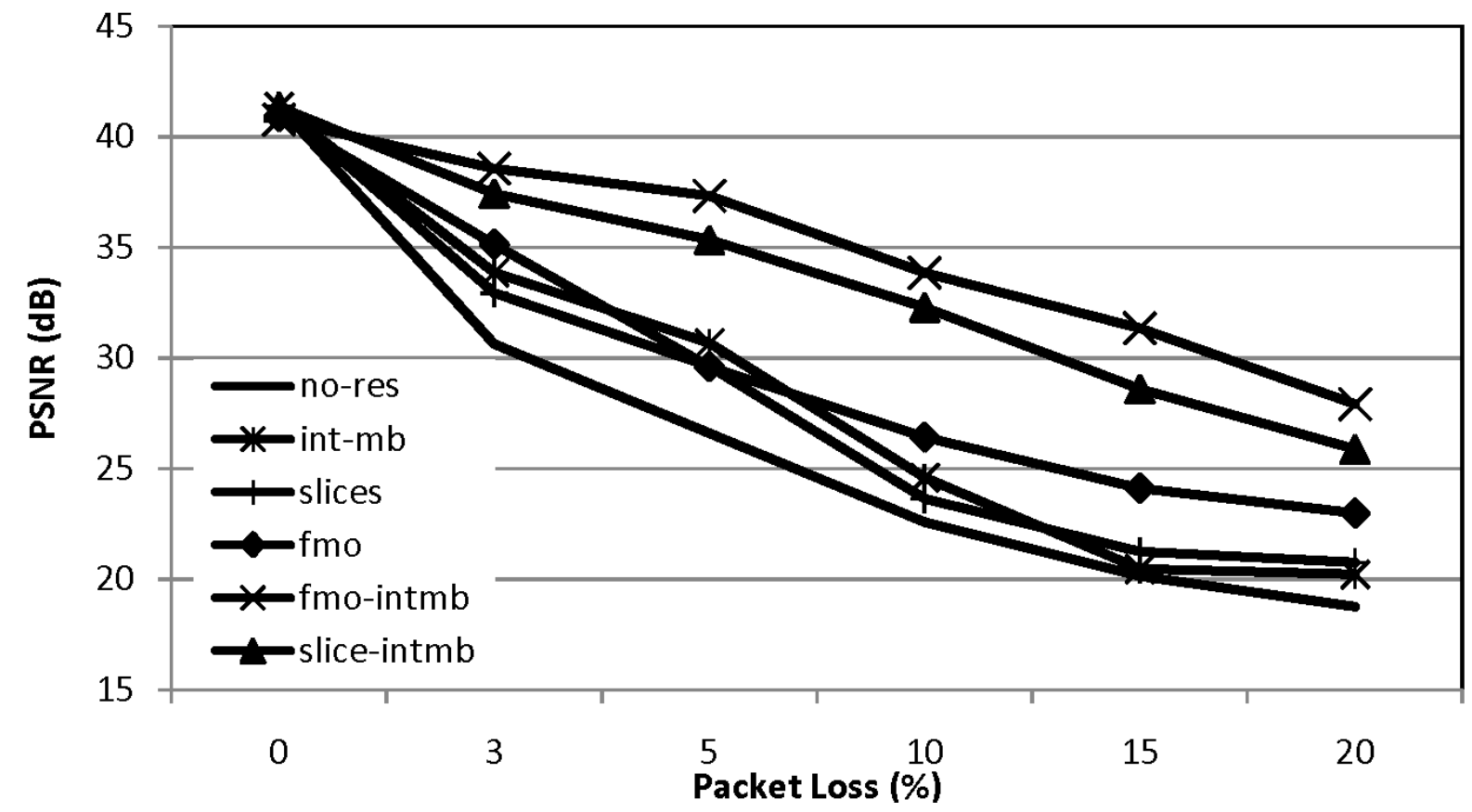

Fig. 2 Video quality with different error resiliency schemes with random losses

In the case when the error resiliency is added, for one technique at a time, the three slices per frame generally results in the worst quality, while the checkerboard FMO results in better quality followed by intra coded macro blocks. When two error resiliency techniques were combined, the two slice checkerboard FMO combined with intra-coded macro blocks has the best performance followed by three slices combined with intra coded macro blocks. Again, ordering at zero packet loss reflects the relative coding overhead from each technique. In general, it is better to combine at least two error resiliency techniques. For random errors, it is important to note that utilizing checkerboard FMO with larger packet sizes is better than smaller packet sizes with more slices.

\subsection{Response of source robustness to burst losses}

In the case of burst errors the packet size has an important effect. Fig. 3 shows that increasing the number of slices results in good quality video up to burst lengths equal to the number of slices in a frame. In these circumstances, the FMO which performed better than the slices for isolated errors has lower PSNR than slices for burst errors of length greater than the number of slices/FMO per frame. The reason behind this is that the initial PSNR of the stream with simple slicing is better than the streams having the same number of FMO slice groups due to lower compression efficiency of the FMO, especially in case of checkerboard. In case of burst errors, it is possible that all 
the slices (or FMO groups) related to the same frame may lost, thereby limiting the effect of the concealment strategy as the error concealment through interpolation is no longer possible. In this case loss concealment from the already available good quality sliced frames or lower quality FMO frames decides the quality of the concealed frame, in which the quality of frame with slices may become equal or even better than the frames with FMO. Figure 3 shows that at longer burst lengths, FMO with two slice groups (denoted as fmo2) is of lower quality than the three slices per frame (denoted as slice3). Similarly FMO with eight slice groups (fmo8) is of approximately the same quality as that of the frames with the same number of slices (slic8) after the burst length 8 . The graph shows frames with sixteen slices have the best quality. It can be noted that comparing the initial PSNR of the streams with the same number of FMO groups and slices (fmo8, slice8), the stream with slices has better compression efficiency and should be preferred. Therefore in case of burst errors simple slicing should be preferred over FMO as it has better quality when there are no errors and approximately equal quality in case of errors.

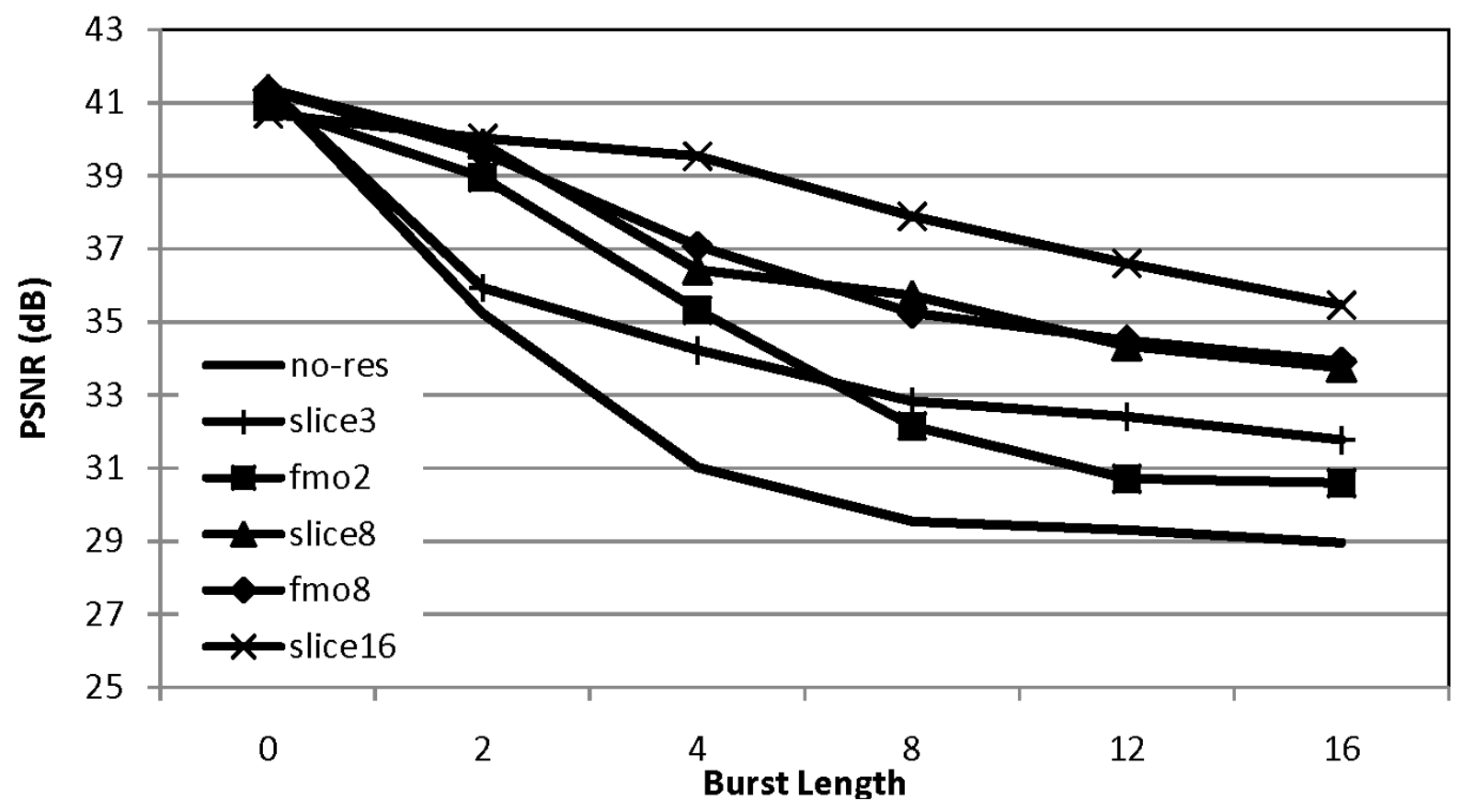

Fig. 3 Video quality with different error resiliency schemes with burst losses

\subsection{Raptor Encoding with losses}

The same video clip (rush hour) was initially encoded at a rate of 4.5 Mega bits per second (Mbps) without applying any source robustness. The video stream was simulated using raptor encoding with a redundancy of $3 \%, 5 \%, 10 \%$, $15 \%, 20 \%, 30 \%$ and $50 \%$ while transmitting over channels with $3 \%, 5 \%, 10 \%, 15 \%$ and $20 \%$ packet losses. To provide room for the redundancy introduced by raptor encoding and hence keeping the transmission rate fixed, the stream that was transmitted with 3\% redundancy was encoded at a rate 3\% less than the stream not providing any protection that is at $4.5 \mathrm{Mbps}$. Similarly the stream transmitted with 5\% redundancy was compressed at a rate 5\% less than stream without protection and so on. Figure 4 shows the PSNR versus packet loss of all the above seven streams and denoted by rate- 3 , rate- 5 and rate- 10 for $3 \%, 5 \%, 10 \%$ redundancy etc.

A keen observation of Fig. 4 shows that the initial PSNR of the streams with less compression due to the smaller amount of redundancy is relatively more than those with more compression, leaving room for redundancy due to raptor encoding. It is also clear that the drop in PSNR with increased packet loss is less for the streams with more compression due to increased protection and redundancy provided by raptor encoding. It is clear that although the drop in quality of rate-50 is less than the drop in quality of rate-30 and rate-20 it still has less PSNR when transmitted through an error prone channel having a packet loss of up to $20 \%$. 
Rate-30 and Rate-50 are compared with the best of source robustness schemes fmo-intmb and slice-intmb for isolated errors and slice-16 for burst errors shown in Fig. 5. It is clear that the raptor encoding provides better results compared to that of source error robustness schemes for isolated errors with packet losses more than $1 \%$ and burst losses more than $5 \%$, specifically in the case of rate-50.

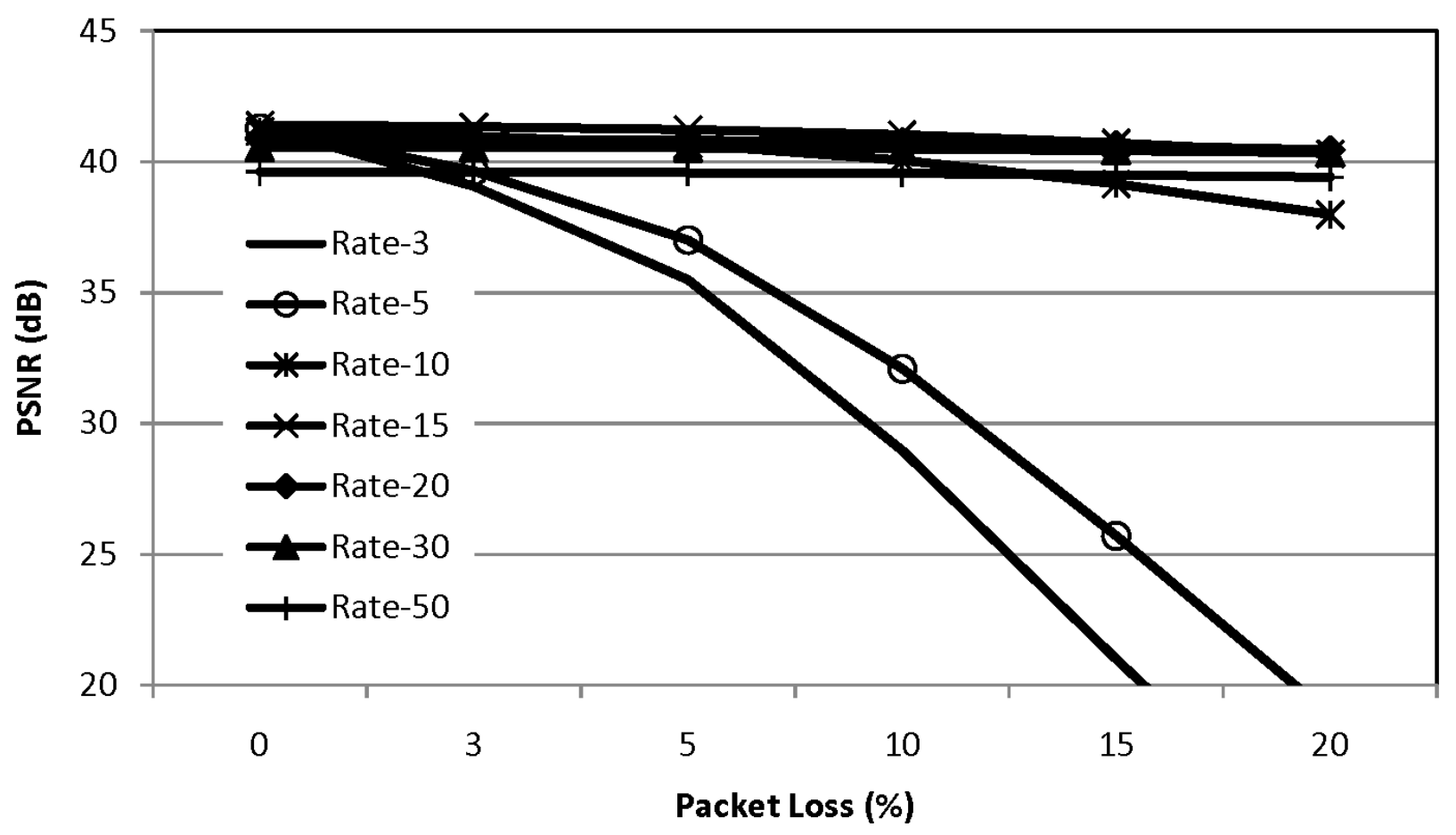

Fig. 4 PSNR Vs packet loss for raptor encoding with different amount of redundancies 


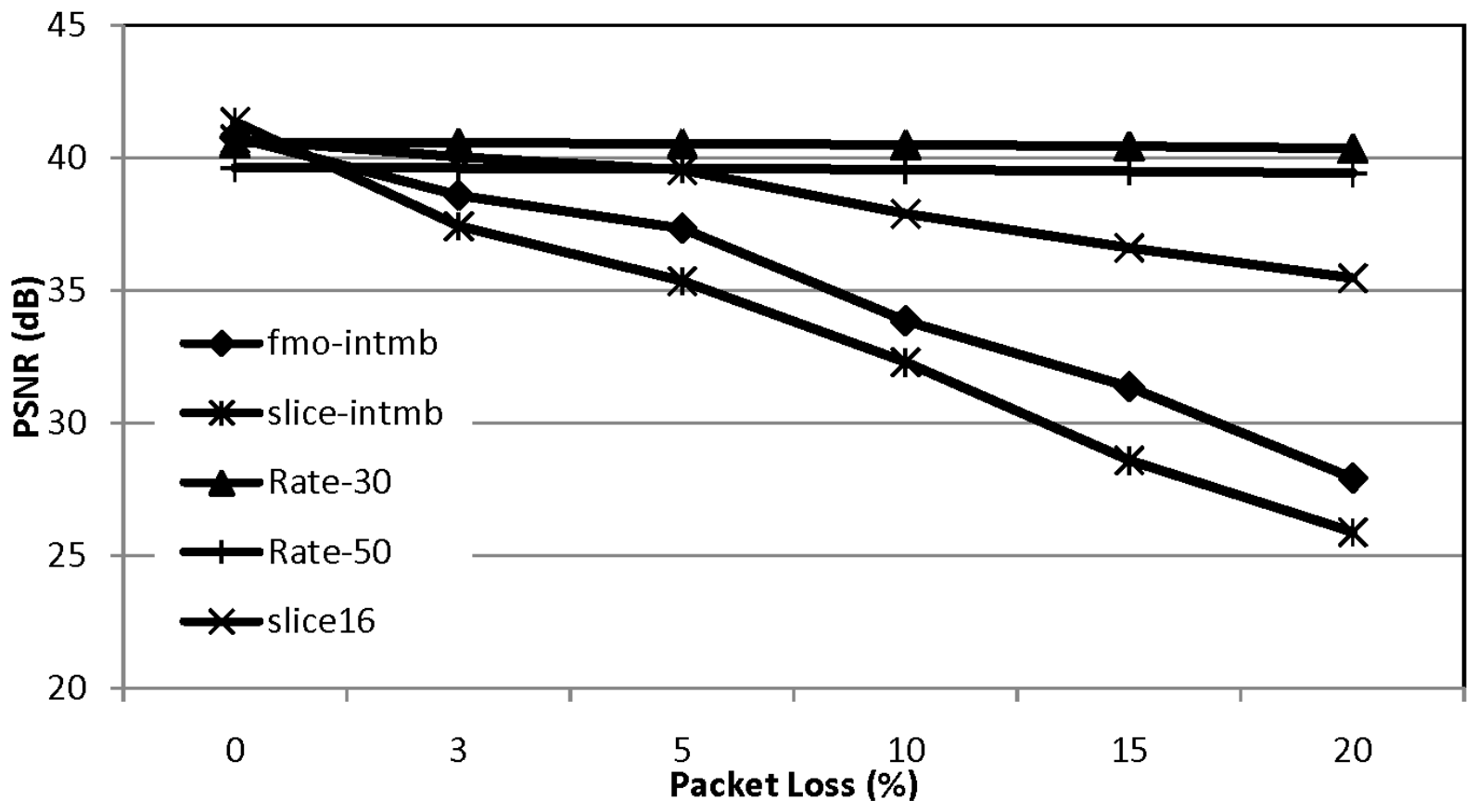

Fig. 5 Comparison between source robust streams and raptor encoded streams

\section{Robust Switching Tests}

4.1 Objective Quality Tests

To carry out robust switching, a feedback channel is considered with DVB-RCS and is shown in Fig. 1. Initially the channel conditions were assumed error free and the video was streamed without any protection in order to benefit from higher quality of non-protected signal. During the period of poor channel conditions, switching to robust

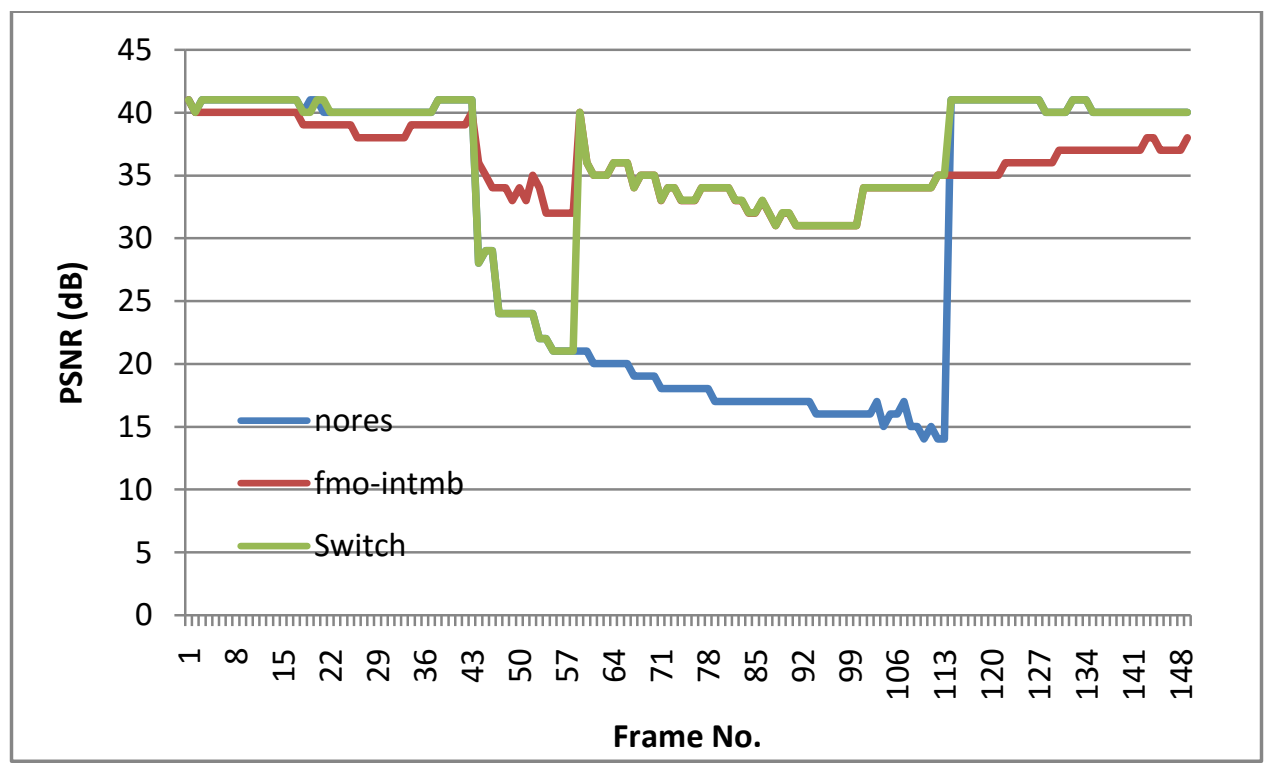

Fig. 6 Video quality for robust switching in a channel with isolated losses

stream is done using switching intra (SI) frame and then back when the packet loss rate is reduced. For simulation purposes, the feedback time threshold before switching back to a non-protected stream was taken to be fifteen frames or $500 \mathrm{~ms}$ for a sequence with $30 \mathrm{fps}$, in order to properly simulate the time taken by satellite feedback 
channel. Although in a practical implementation switching can be initiated at any location using a switching frame. The frame structure was taken as IPPPPP with SI frames at fixed locations instead of intra frames. At the switching instant an SI frame is transmitted followed by the frames of the new stream.

To analyze the effect of switching between the streams in the presence of randomly occurring bit errors, a comparison was made between: (1) streaming solely with no error resilience, (2) streaming with the best of the schemes in Fig. 2, that is FMO with intra macro block refresh, and 3) switching (Switch in Fig. 6) at the start of poor channel conditions to option (2) with error resiliency. A period of poor channel conditions with a rate of $10 \%$ random packet losses was created.

To compare results the same pattern of losses was replicated for all three streams. In Fig. 6, the graph is divided into three regions: (1) from fame 0 to frame 49, when there are no losses, (2) after frame 50 when random packet losses start to occur, and (3) after frame 100 when again better channel conditions without losses occurs. In the case of switching, an SI frame was used in transition between the streams. However, the bit rate for all three streams was the same. In fact, the first packet loss actually occurred at frame 45 , but switching occurred only at frame 60 due to the feedback delay and presence of a switching frame at that location.

Prior to frame 45, the video quality plots in Fig. 6 for no resilience and switching are identical with video quality declining once the effect of the initial intra frame has faded. Using error resiliency during this period results in worst coding efficiency because of the overhead involved in providing robustness. When the first packet loss occurs, both the no resiliency and switching curves drop in quality, but as a result of the SI frame at frame position 60, the video quality of the switched stream recovers. The decoder is resynchronized as a result of the SI frame and as a result the quality becomes equivalent to the protected stream without switching. On entering the "good" period after frame 100 , an SI frame resynchronizes the decoder for all the three sequences at the switching instant at frame 115. However, the average quality of the switched stream is better than the other two.

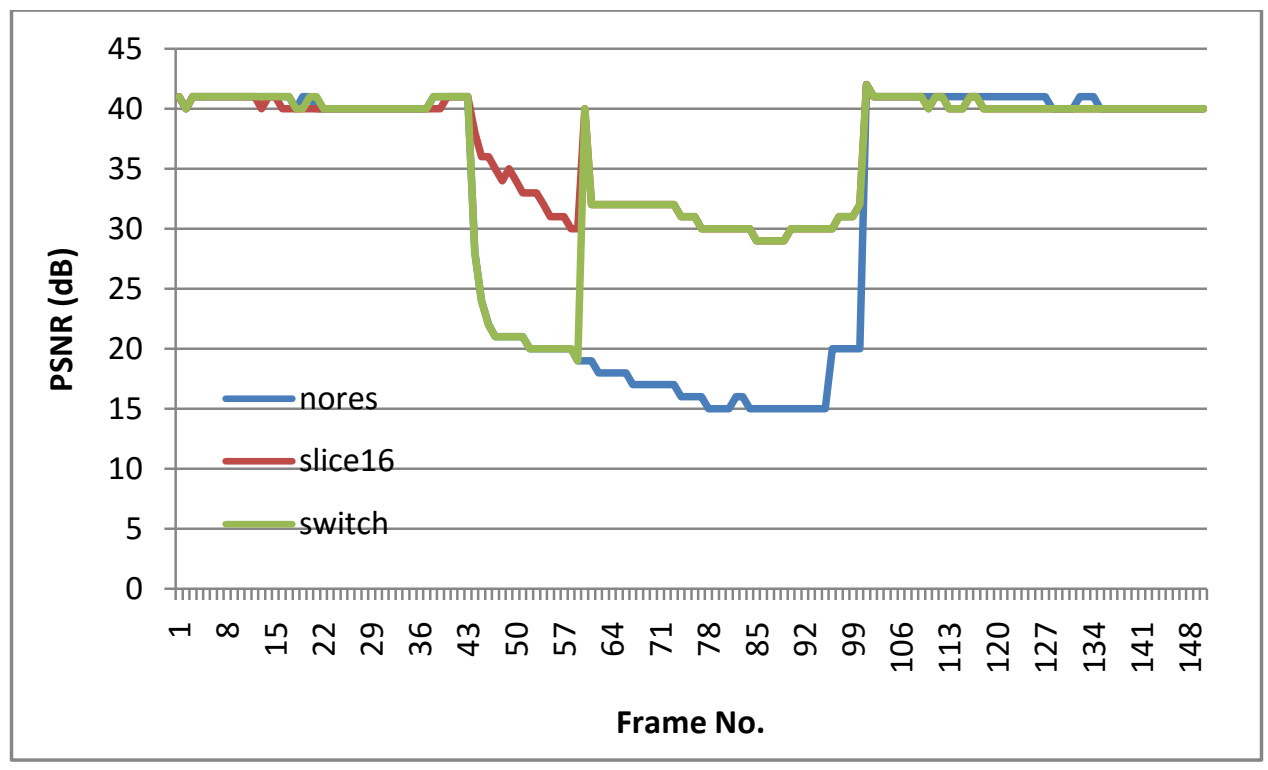

Fig. 7 Video quality for robust switching in a channel with burst losses

Table 1 Comparison of mean PSNR between robust switching and other schemes for various test video sequences

\begin{tabular}{|l|c|c|c|c|}
\hline Stream & $\begin{array}{l}\text { Blue Sky } \\
(42-115)\end{array}$ & $\begin{array}{l}\text { River Bed } \\
(50-110)\end{array}$ & $\begin{array}{l}\text { Tractor (60- } \\
140)\end{array}$ & $\begin{array}{l}\text { Rush Hour } \\
(70-139)\end{array}$ \\
\hline No-res (Random) & 27.42 & 25.72 & 26.06 & 31.00 \\
\hline $\begin{array}{l}\text { FMO MB } \\
\text { (Random) }\end{array}$ & 28.4 & 24.01 & 27.62 & 35.53 \\
\hline $\begin{array}{l}\text { Switched } \\
\text { (Random) }\end{array}$ & 32.53 & 28.11 & 30.41 & 36.77 \\
\hline
\end{tabular}




\begin{tabular}{|l|c|c|c|c|}
\hline No-res (Burst) & 18.26 & 20.71 & 19.93 & 24.00 \\
\hline Slice16 (Burst) & 32.53 & 26.78 & 30.67 & 35.78 \\
\hline Switched (Burst) & 36.72 & 32.53 & 33.56 & 37.08 \\
\hline
\end{tabular}

Figure 7 is a comparison of switching in the presence of burst errors. During the poor channel period between frames 45 and 100 two packet burst errors of length four were introduced. Similar to the random error tests, three schemes were compared. However, error resiliency was through the best of the schemes in Fig. 3, which is through simple slicing with 16 slices. It is shown that the robust switched scheme is superior throughout the session, while the no error resiliency scheme fails to recover from lost packets during the period of poor channel conditions. Therefore, packet loss bursts can cause a breakdown in video quality if intra updates due to SI frames are not used. The increase in quality at switching instants is due to the SI frames that confines the drift error.

Similarly, confining the raptor encoding to period of poor channel conditions will not only increase the PSNR in the presence of packet loss but will also reduce the high computation required by the raptor encoding as shown in Fig. 8 . During the period of good channel conditions the stream is transmitted without applying raptor encoding. On reception of packet loss interrupt, the video is streamed using raptor encoding and vice versa. Here again the poor channel condition is shown between frames 45 and 100 similar to the previous two cases. It is important to note that the video stream transmitted using raptor encoding (rate-50) and shown in Fig. 8 is compressed at a rate 50 percent less than the stream without protection.

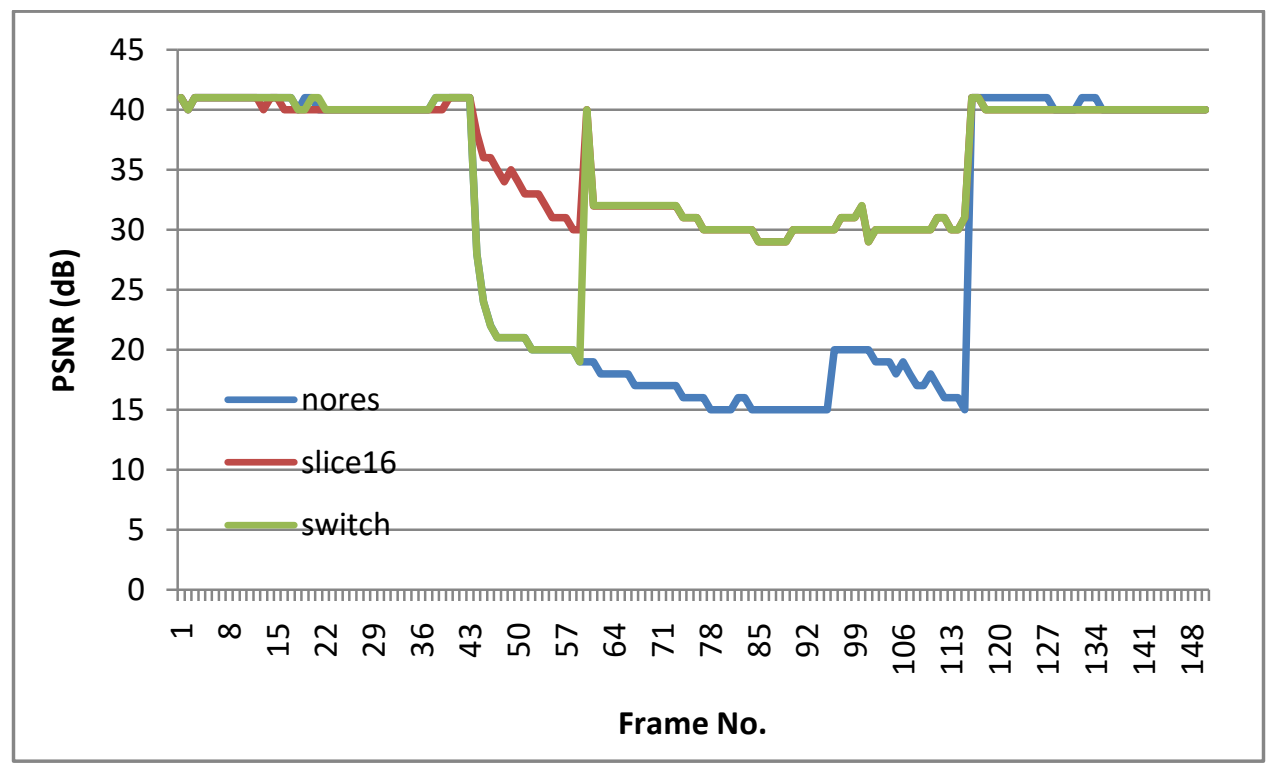

Fig. 8 Video quality for switching to raptor encoded stream

Thus the rate-50 has lower quality and requires an SI frame to switch to it without introducing any drift error.

Table 1 summarizes a set of extensive tests in which poor channel conditions, random and burst errors, were simulated for different test videos, blue sky, river bed, tractor and "rush hour. The frame numbers at which switching occurs is provided in brackets in the first row along with the names of the test sequences. The PSNRs in the third and the last row highlight the advantage of the robust switching scheme, which is up to 3-4 dB in PSNR compared to using a robust scheme without switching for entire session.

4.2 Subjective Quality tests

Perceptual quality measurement or quality of enhancement deals with the quality of video as perceived by human 
eyes. In this test, videos with and without switching were shown to 21 different viewers and were asked to score the quality on a scale of 1 to 5 with 5 the highest and 1 the lowest quality. The average values of the mean opinion score (MOS) are given in table 2, 3 and 4 for videos with switching to robust streams, without switching to robust stream and video streams with high robustness respectively. All the video clips are 30 minutes long, the frequency of switching to and from robust stream is given in table 2 under the heading of "Switching Frequency" with values of $10,20,30$ and 50. A switching frequency of 10 means that the whole video transmission time is divided in to 10 time slots with five good slots assuming no packet loss and 5 bad time slots with different loss rates. The transmission is started assuming good time slot and without any robustness, at the arrival of bad time slot the video is switched to high robust stream and so on. The concept is shown in figure 9 for a switching frequency of 10 . In figure 9, the upper dotted line shows stream with robustness and the lower dotted line shows stream without robustness. The vertical lines divide the time into different time slots designated with good and bad time slots. The arrows show the original transmission of the steam. The arrows with upward directions show switching to the robust stream while the arrows with downward directions show switching back to stream without any protection. Table 3 shows the same video sequences subject to same packet loss conditions but without switching to high robust streams while table 4 gives video streams with high robustness and subjected to same packet loss conditions as the other two. The column with "switching Frequency" in table 3and 4, is only for comparison with table 2 and is not an indication of actual switching.

Stream with

robustness

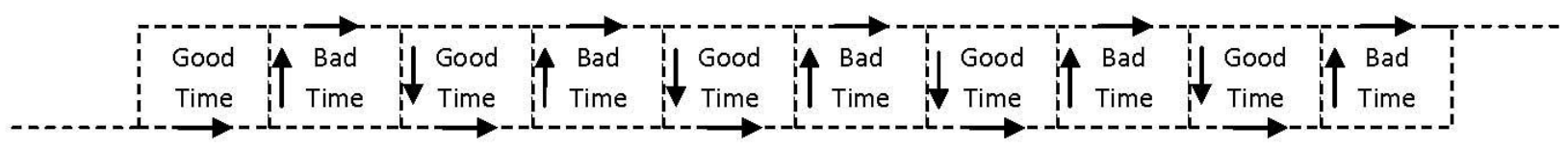

Stream

without

Fig 9. Stream switching with switching frequency of 10

The analysis shows that if switching frequency is increased above 30 for a video of 30 minutes play time that is switching once per minute on average, the quality reduces considerably although the objective quality in terms of PSNR is much better. The video quality as shown in table 3 is much lower as compared to the quality of streams with switching facility given in table 2 and the quality of streams with high robustness only given in table 4 . The results show that keeping switching frequency less than once per minute on average is acceptable as compared to streams without switching as shown in table 3 and 4 . Video with switching frequency of 20 that is switching once per 1.5 minutes on average is on the higher end and increases with decrease in switching frequency. Therefore it is required to limit this frequency to once per 1.5 on the average.

Table 2: Quality of Enhancement of video streams with switching

\begin{tabular}{|l|r|r|r|r|r|}
\hline $\begin{array}{l}\text { Switching } \\
\text { Frequency }\end{array}$ & $\begin{array}{c}\text { Clip 1 } \\
\text { (MOS) }\end{array}$ & $\begin{array}{c}\text { Clip 2 } \\
\text { (MOS) }\end{array}$ & $\begin{array}{c}\text { Clip 3 } \\
\text { (MOS) }\end{array}$ & $\begin{array}{c}\text { Clip 4 } \\
\text { (MOS) }\end{array}$ & $\begin{array}{c}\text { Clip 5 } \\
\text { (MOS) }\end{array}$ \\
\hline $\mathbf{1 0}$ & 4.4 & 4.2 & 4.8 & 4.2 & 4.4 \\
\hline $\mathbf{2 0}$ & 3.4 & 3.6 & 3.6 & 3.4 & 3.4 \\
\hline $\mathbf{3 0}$ & 3 & 3 & 3 & 3 & 3 \\
\hline $\mathbf{5 0}$ & 1.6 & 1.6 & 1.8 & 2 & 2.2 \\
\hline
\end{tabular}

Table 3: Quality of Enhancement for video streams without robustness (No switching)

\begin{tabular}{|l|r|r|r|r|r|}
\hline $\begin{array}{l}\text { Switching } \\
\text { Frequency }\end{array}$ & $\begin{array}{r}\text { Clip 1 } \\
\text { (MOS) }\end{array}$ & $\begin{array}{c}\text { Clip 2 } \\
\text { (MOS) }\end{array}$ & $\begin{array}{c}\text { Clip 3 } \\
\text { (MOS) }\end{array}$ & $\begin{array}{c}\text { Clip 4 } \\
\text { (MOS) }\end{array}$ & $\begin{array}{r}\text { Clip 5 } \\
\text { (MOS) }\end{array}$ \\
\hline 10 & 2.2 & 2.6 & 2.2 & 2.6 & 2.4 \\
\hline
\end{tabular}




\begin{tabular}{|l|r|r|r|r|r|}
\hline $\mathbf{2 0}$ & 1.75 & 1.4 & 1.4 & 1.75 & 1.8 \\
\hline $\mathbf{3 0}$ & 1 & 1 & 1 & 1 & 1 \\
\hline $\mathbf{5 0}$ & 1 & 1 & 1 & 1 & 1 \\
\hline
\end{tabular}

Table 4: Quality of Enhancement for robust video streams only (No switching)

\begin{tabular}{|l|r|r|r|r|r|}
\hline $\begin{array}{l}\text { Switching } \\
\text { Frequency }\end{array}$ & $\begin{array}{c}\text { Clip 1 } \\
\text { (MOS) }\end{array}$ & $\begin{array}{c}\text { Clip 2 } \\
\text { (MOS) }\end{array}$ & $\begin{array}{c}\text { Clip 3 } \\
\text { (MOS) }\end{array}$ & $\begin{array}{c}\text { Clip 4 } \\
\text { (MOS) }\end{array}$ & $\begin{array}{c}\text { Clip 5 } \\
\text { (MOS) }\end{array}$ \\
\hline $\mathbf{1 0}$ & 4 & 4 & 4 & 4 & 4 \\
\hline $\mathbf{2 0}$ & 3.2 & 3.1 & 3.2 & 2.9 & 3.0 \\
\hline $\mathbf{3 0}$ & 3 & 3 & 3 & 3 & 3 \\
\hline $\mathbf{5 0}$ & 1.5 & 1.3 & 1.8 & 1.8 & 2 \\
\hline
\end{tabular}

\section{Conclusion}

In this paper different error resiliency schemes of the H.264 were compared with the raptor encoding for video streaming over wireless channels. Source error resilience schemes were first compared with one another and it was observed that the Flexible Macro block Ordering combined with intra macro block update behaved better in the case of random losses while increased slicing was the best protection against burst losses. The drop in quality due to redundancy that arose from introducing source robustness and raptor encoding in the presence and absence of packet loss was also studied. It was shown that for packet losses lower than $1 \%$ the source robustness overcame the raptor encoding scheme in the case of random losses while for higher error rates the raptor outperformed source robustness. In the case of burst losses the source robustness outperformed the raptor code for losses less than 5\% while at higher rates the raptor code proved to be more efficient. It was explained if the video stream was compressed such that room was provided to introduce redundancy due to raptor encoding (to keep the transmission rate at minimum), then the PSNR at $20 \%$ and $30 \%$ redundancy outperformed the $50 \%$ redundancy because of the higher initial PSNR. In the second step SI frames were used to switch between streams with different degrees of robustness. It was shown that better video quality can be achieved if the stream without error resiliency is used for the time when the channel is error free and robust streaming is limited to the time slots when the channel is error prone. Feedback messages were used to indicate the channel state and SI frames were used to confine the ongoing drift errors before starting a new stream with new error resilience features. The video streams with and without switching were assessed for quality of enhancement by different viewers. In this test it was shown that switching between high robustness streams and stream without protection is better as compared to using one stream for the whole session irrespective of loss rate. But this too should be done intelligently as frequent switching can improve the PSNR but reduces the perceptual quality and can results in an annoying effect. The results show that if the switching frequency is restricted to only 20 percent, that is a minimum of 1.5 minutes gap between the two switching instances will not only improve the PSNR but also the perceptual quality. Overall it was shown that an increase of 3-4 $\mathrm{dB}$ in the average quality of stream could be achieved.

\section{References}

[1]. H. Skinnemoen, R. Leirvik, J. Hetland, H. Fanebust, V. Paxal (2004) Interactive IP-Network Via Satellite DVB-RCS. IEEE Journal on selected areas in communications 22(3): 508-517

[2]. O. Alphand, P. Berthou, T. Gayraud, S. Combes (2005) QoS architecture over DVB-RCS satellite networks in a NGN framework. proceedings of IEEE globcom2005

[3]. J. Neale, R. Green, J. Landovskis (2004) Interactive channel for multimedia satellite net- works. IEEE communication magazine 39(3): 192-198 
[4]. A. Donner, S. Bovelli, S. Shabdanov (2002) Reliable multicast based on DVB-RCS. 20th AIAA international communication satellite systems conference and exhibit

[5]. G. Gardikis, N. Zotos., A. Kourtis (2009) Satellite media broadcast with adaptive coding and modulation. International journal of digital multimedia broadcasting: 1-10

[6]. A. Gotta, P. Barsocchi (2008) Experimental video broadcasting in DVB-RCS/S2 with land mobile satellite channel: a reliability issue. IEEE International workshop on satellite and space communications: 234-238

[7]. M. Ghanbari (2003) Standard Codecs: Image Compression to Advanced Video Coding. Stevenage IET Press, UK

[8]. T. Stockhammer, M.M. Hannuksela, T. Wiegand (2003) H.264/AVC in wireless environ- ments. IEEE Transactions on Circuits and Systems for Video Technology 13 (7): 657-673

[9]. S. Kumar, L. Xu, M.K. Mandal, S. Panchanathan (2003), Error resiliency schemes in H.264/AVC standard. Elsevier J. of Visual Communication and Image Representation (Spe- cial issue on Emerging H.264/AVC Video Coding Standard) 17 (2): 1-26

[10]. Nikolaos Thomos, Rethnakaran Pulikkoonattu, Pascal Frossard, Intermediate Performance Analysis of Growth Codes arXiv:1211.4014 [cs.IT]

[11]. A. Shokrollahi (2006) Raptor codes. IEEE Transactions on Information Theory 52(6): 2551-2567

[12]. M. Luby (2002) LT codes. in Proc. of the 43rd Annual IEEE Symposium on Foundations of Computer Science: $271-280$

[13]. 3GPP TS 26.346 V7.4.0. (2007) Technical specification group services and system aspects; multimedia broadcast/multicast service; protocols and codecs

[14]. ETSI EN 302304 (2004) Digital Video Broadcasting (DVB): Transmission Sys- tem for Handheld Terminals (DVB-H) European Telecom-munication Standard, (2004) http://www.dvb-honline.org/technology

[15]. Shakeel Ahmad, Raouf Hamzaoui, Marwan M. Al-Akaidi (2011), Unequal Error Protection Using Fountain Codes WithApplications to Video Communication. IEEE Transactions on Multimedia 13(1): 92-120

[16]. T. Schierl, K. Ganger, C. Hellge (2006) Svc-based multisource streaming for robust video transmission in mobile ad hoc networks. IEEE Wireless Communications 13(5): 96-103

[17]. C. Hellge, D. Gomez-Barquero, T. Schierl, T. Wie-gand (2011) Layer-aware forward error correction for mobile broadcast of layered media. IEEE Transactions on Multimedia 13 (3): 551-562

[18]. B.S. Choi, D. Y. Suh (2009) Peer-to-peer scalable video streaming using Raptor code. in Proc. of 1st IEEE International Conference on Ubiquitous and Future Networks: 137-141

[19]. R. Bernardini, M. Durigon, R R. Rinaldo, A. Vitali (2005) Comparison between multiple description and single description video coding with forward error correc-tion. in Proc. of 7th IEEE Workshop on Multimedia Signal Processing: 1-4

[20]. C. Soldani, G. Leduc, F. Verdicchio, A. Munteanu (2006) Multiple description coding versus transport layer fec for resilient video transmission. in Proc. of International Conference on Digital Telecommunications

[21]. S. Wenger (2003) H.264/AVC over IP. IEEE Transactions on Circuits and Systems for Video Technology $13(7): 645-656$

[22]. J. Osterman, J. Bormans, P. List, D. Marpe, M Narroschke, F. Pereira, T. Stockhammer, T. Wiegand (2004) Video coding with H.264/AVC: Tools, performance and complexity. IEEE Circuits and Systems Magazine: 7-28 
[23]. N. Son, S. Jeong (2008) An Effective Error Concealment for H.264/AVC. IEEE 8th Inter- national Conference on Computer and Information Technology Workshops: 385-390

[24]. R. K. Karczewicz (2003) The SP- and SI-frames design for H.264/AVC. IEEE Transactions on Circuits and Systems for Video Technology 13 (7): 637-644

[25]. T. Stockhammer, W. Zia (2007) Error-resilient coding and decoding strategies for video communication: P. A. Chou and M. van der Schaar, Eds. Multimedia in IP and Wireless Networks, Academic Press, Burlington, MA, 13-58, July 2007

[26]. V. Vars, M. N. Hannuksela (2001) Non-normative error concealment algorithms. ITU-T SGI6 Doc., VCEG-N62

[27]. Qualcomm Raptor Technology - Forward Error Correction,

[28]. J. W. Byers, M. Luby, M. Mitzenmacher (2002) A digital fountain approach to asyn- chronous reliable multicast. IEEE Journal of Selected Areas in Communication 20 (8): 1528- 1540

[29]. A. Shokrollahi (2004) Raptor Codes. In Proc. IEEE International Symposium on Infor- mation Theory

[30]. Pasquale Cataldi, Miquel Pedros Shatarski, Marco Grangetto, Enrico Magli (2006) Imple- mentation and performance evaluation of LT and Raptor codes for multimedia applications. International Conference on Intelligent Information Hiding and Multimedia Signal Process- ing: 263-266

[31]. H. Kushwaha, Y. Xing, R. Chandramouli, H. Heffes (2008) Reliable Multimedia Transmis- sion Over Cognitive Radio Networks Using Fountain Codes. in proc. IEEE 96 (1): 155-165

[32]. Michael Luby, Tiago Gasiba, Thomas Stockhammer, Mark Watson (2007) Reliable Mul- timedia Download Delivery in Cellular Broadcast Networks. IEEE Transactions on broad- casting 53 (1): $235-246$

[33]. Avani U Pandya, Sameer D Trapasiya, Santhi S Chinnam (2013) Performance analysis of AL-FEC raptor code over 3GPP EMBS networks. International journal of renewable energy technology 2 (4): 601-608

[34]. Why digital fountain raptor code is better than reed-solomon erasure codes for streaming applications, white paper, copy right (c) 2005, digital foundation, Inc.

[35]. M. Altaf, M. Fleury and M. Ghanbari (2011) Resilient Video Stream Switching for Mobile Wireless Channels. Journal on Mobile Multimedia (JMM) 7 (3): 216-235

[36]. Intelligent Streaming, https://technet.microsoft.com/en-us/library/cc732815.aspx

[37]. Streaming media basics, http://service.real.com/help/library/guides/helixproducer/htmfiles/realsyst.htm

[38]. P. Chatziparaskevas, G. Koltsidas, F.-N. Pavlidou (2011) on the fairness of return channel capacity allocation in DVB-RCS-based satellite networks. International journal of satellite communication and networking, 29: 163-184

[39]. Harald Skinnemoen, Christian Rigal, Ana Yun, Lars Erup, Nader Alagha, Alberto Ginesi (2013) DVBRCS2 overview. international journal of satellite 